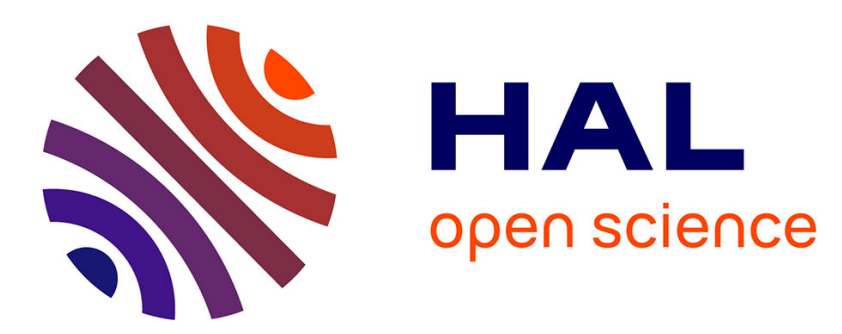

\title{
Étude expérimentale et théorique de l'amplification gazeuse dans un compteur proportionnel cylindrique à basse pression
}

\author{
M. Cukier, P. Dhez, P. Jaegle
}

\section{- To cite this version:}

M. Cukier, P. Dhez, P. Jaegle. Étude expérimentale et théorique de l'amplification gazeuse dans un compteur proportionnel cylindrique à basse pression. Revue de Physique Appliquée, 1972, 7 (4), pp.357-365. 10.1051/rphysap:0197200704035700 . jpa-00243639

\section{HAL Id: jpa-00243639 \\ https://hal.science/jpa-00243639}

Submitted on 1 Jan 1972

HAL is a multi-disciplinary open access archive for the deposit and dissemination of scientific research documents, whether they are published or not. The documents may come from teaching and research institutions in France or abroad, or from public or private research centers.
L'archive ouverte pluridisciplinaire HAL, est destinée au dépôt et à la diffusion de documents scientifiques de niveau recherche, publiés ou non, émanant des établissements d'enseignement et de recherche français ou étrangers, des laboratoires publics ou privés. 
Classification

Physics Abstracts

$11.10,14.40,10.20$

\title{
ÉTUDE EXPÉRIMENTALE ET THÉORIQUE DE L'AMPLIFICATION GAZEUSE DANS UN COMPTEUR PROPORTIONNEL CYLINDRIQUE A BASSE PRESSION
}

\author{
M. CUKIER, P. DHEZ et P. JAEGLE \\ Laboratoire de Chimie Physique « Matière et Rayonnement » Associé au CNRS \\ Université de Paris VI, Bâtiment 350, Centre d'Orsay, 91-Orsay
}

(Reçu le 7 mars 1972, révisé le 25 mai 1972)

\begin{abstract}
Résumé. - Ce travail a pour but l'utilisation dans les conditions optimales d'un compteur proportionnel cylindrique comme détecteur pour des rayonnements peu énergétiques du domaine $\mathrm{X}$ ultra mou.

Nous étudions expérimentalement l'amplification gazeuse jusqu'à une valeur de $10^{3}$, pour des pressions de méthane inférieures à une centaine de torrs et des tensions électriques de l'ordre du millier de volts. Nous présentons un certain nombre de remarques concernant le fonctionnement du compteur en intégrateur de flux.

Aux basses pressions, les formules analytiques usuelles de l'amplification sont inutilisables. Nous simulons donc en ordinateur une avalanche électronique à partir d'hypothèses physiques simples. Ce calcul qui utilise trois paramètres ajustables dont on peut donner une interprétation physique, rend compte de l'ensemble des résultats expérimentaux.

Abstract. - The purpose of this work is the study of the optimal efficiency of a cylindrical proportional counter used as a detector for soft $\mathrm{X}$ ray radiation.

We study the experimental gas gain until the gas amplification coefficient reaches the value $10^{3}$ for a methane pressure lower than one hundred torrs and a counter voltage of about one thousand volts. We explain how the counter can work as an integrating flow detector.

At low pressures, the usual analytical gas amplification formulas are not available. Hence, using a simple physical assumption we perform calculations which simulate an electronic avalanche. The three ajustable parameters used in this method can be given a physical interpretation.
\end{abstract}

L'intérêt de l'utilisation des compteurs proportionnels à gaz comme détecteurs de rayonnement dans le domaine $\mathrm{X}$ mou et ultraviolet extrême a été largement démontré [1], [2], [3]. Ces compteurs ont pour avantage dans un domaine étendu de longueurs d'onde, de délivrer des signaux proportionnels à l'énergie reçue. De plus, ils se prêtent à des prévisions théoriques pour la création des paires ion-électron primaires comme pour l'amplification par avalanche gazeuse des charges initiales.

Cependant leur emploi avec des rayonnements faiblement ionisants fait apparaître des problèmes nouveaux. D'une part l'amplification gazeuse doit être grande - elle peut atteindre $10^{5}$-, d'autre part il se révèle fréquemment avantageux d'utiliser le compteur non pas en tant que tel mais comme intégrateur de flux. Ceci résulte en partie de la nature du rayonnement, puisque l'ionisation primaire est faible et que l'intégration du flux permet en certains cas d'éviter les inconvénients d'une amplification trop élevée, mais aussi du fait que plusieurs types de

(*) Associé au C. N. R. S. source d'ultraviolet extrême fournissent une émission pulsée dont l'intensité maximale dépasse la capacité de comptage du détecteur.

L'amplification gazeuse dépend du champ électrique $E$ et de la pression $p$ dans le compteur, plus précisément du rapport $E / p$ [4]. Les conditions générales qui doivent être réalisées pour obtenir une grande amplification ont été précisées [1], [2]. Il est en général nécessaire de travailler avec une pression nettement inférieure à la pression atmosphérique. Mais alors que l'amplification a été très étudiée en fonction de la tension électrique [2], [5]-[9], sa variation en fonction de la pression gazeuse est moins bien connue. Des formules analytiques (formules de Korff, Diethorn, Curran...) rendent compte des résultats expérimentaux dans les conditions les plus usuelles (pression voisine de la pression atmosphérique, tension de l'ordre du millier de volt), mais elles ne sont pas valables pour les basses pressions ni pour les valeurs élevées de $E / p$. Par conséquent l'un des buts de ce travail est de rendre compte théoriquement des variations de l'amplification aux basses pressions gazeuses. Pour cela nous avons établi un modèle d'avalanche électronique 
calculable en ordinateur. Le calcul est effectué en l'absence de charge d'espace et le cas traité pratiquement est celui d'un compteur rempli de méthane.

Pour étudier expérimentalement la variation de l'amplification en fonction de la pression nous avons utilisé le rayonnement émis dans l'ultraviolet extrême par le plasma obtenu en focalisant le faisceau d'un laser de puissance sur une cible d'aluminium [10]. Le compteur recevait donc un rayonnement pulsé et fonctionnait en intégrateur de flux. En décrivant la méthode expérimentale nous serons donc amenés à présenter quelques remarques sur ce mode de fonctionnement des compteurs.

Nous avons choisi le méthane comme gaz de remplissage pour deux sortes de raisons. Ce gaz est l'un de ceux pour lesquels le plus de données physiques ont été publiées, notamment en ce qui concerne les sections efficaces d'interaction par choc électron-molécule. D'autre part les gaz monoatomiques et diatomiques (par exemple les gaz rares et l'hydrogène) émettent au cours de l'avalanche un rayonnement ultraviolet qui n'est pas complètement réabsorbé et donne lieu à un effet photoélectrique relativement important sur la cathode [5]. Le calcul de l'amplification gazeuse devient alors très complexe. Avec le méthane pur ce phénomène est négligeable dans un domaine étendu de pression et de tension ce qui permet un calcul à la fois plus simple et plus rigoureux.

I. Etude expérimentale de l'amplification gazeuse en fonction de la pression. - Le compteur que nous utilisons pour cette étude est un compteur cylindrique de $18 \mathrm{~mm}$ de diamètre et de $47 \mathrm{~mm}$ de hauteur [1], [3], [11]. L'anode est constituée par un fil de tungstène poli électrolytiquement de $0,06 \mathrm{~mm}$ de diamètre. Il a été montré que le polissage du fil permet de minimiser les fluctuations statistiques de l'amplification gazeuse [12]. La fenêtre d'entrée du rayonnement a la forme d'une fente de $0,5 \mathrm{~mm}$ de largeur et de $10 \mathrm{~mm}$ de hauteur, parallèle à l'anode. Elle est fermée par une membrane de collodion de $1000 \AA$ d'épaisseur qui absorbe relativement peu dans l'ultraviolet extrême. Le schéma du montage électrique est représenté figure 1.

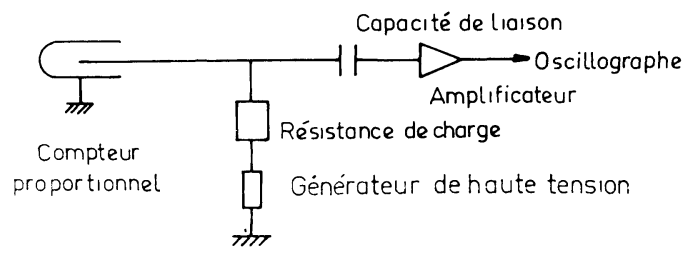

FIG. 1. - Montage coque à la masse.

D'une étude [2] effectuée entre $67 \AA$ et $190 \AA$ pour un taux de comptage de 1000 impulsions par seconde, il résulte que le plateau du compteur s'étend de $1300 \mathrm{~V}$ à $1500 \mathrm{~V}$ pour une pression de 60 torrs de méthane.

Le compteur est monté à la sortie d'un spectrographe à réseaux sous incidence rasante travaillant sous bon vide. Ce spectrographe possède deux réseaux successifs ce qui permet d'éviter le recouvrement des ordres d'interférence dans le spectre analysé [13]. Ce fait a son importance pour la mesure de l'amplification. En effet dans le cas d'une mauvaise séparation des ordres, le compteur recevrait simultanément des photons d'énergie $h v, 2 h v, \ldots$ ce qui rendrait les résultats difficilement interprétables.

La source de rayonnement, décrite en détail par ailleurs [10], est une vapeur métallique portée à très haute température par le faisceau d'un laser à verre dopé au néodyme d'une puissance d'environ $100 \mathrm{MW}$. Les impulsions de rayonnement ont une durée comparable à celle de l'éclair du laser, c'est-à-dire quelques dizaines de nanosecondes.

A) LE COMPTEUR UTILISÉ COMME INTÉGRATEUR DE FLUX. - Dans l'ultraviolet extrême les photons ont une énergie de quelques dizaines d'électrons-volts et ne produisent qu'un petit nombre de paires primaires électron-ion dans le gaz du compteur. Il est donc intéressant, lorsque cela est possible, d'utiliser le compteur en intégrateur et on y est naturellement conduit lorsque les impulsions lumineuses ont une durée inférieure au temps de résolution du compteur qui est d'environ $10^{-6}$ seconde. Ce temps, en moyenne nécessaire pour que les ions créés au cours de l'avalanche quittent la zone étroite où se produit l'amplification, fixe en première approximation le taux de comptage maximal. Si les impulsions lumineuses ont une durée supérieure au temps de résolution, le compteur peut encore fonctionner en intégrateur à condition d'augmenter la constante de temps RC du circuit ; les charges seront alors intégrées pendant un temps de l'ordre de RC.

Le fonctionnement en intégrateur a des conséquences pour le régime d'amplification. Il convient ici de distinguer deux cas.

a) Impulsions lumineuses brèves séparées par un intervalle de temps très supérieur au tempis de résolution du compteur. - Dans ce cas, qui se présente notamment avec l'émission d'un plasma-laser, la durée des impulsions est inférieure au temps de transit des électrons dans le compteur, c'est-à-dire une centaine de nanosecondes pour notre compteur rempli sous une pression de 100 torrs ou moins. Le compteur réagit comme si l'ionisation primaire avait été créée quasi instantanément par une particule unique très ionisante qui fournirait le même nombre de paires que l'impulsion de rayonnement. On mesure une impulsion de tension proportionnelle au nombre total de photons reçus.

Mais pour des impulsions lumineuses de haute intensité l'amplification gazeuse peut être perturbée, comme pour des particules très ionisantes, par la charge d'espace créée par un nombre d'électrons trop élevé dans la zone d'amplification, près de l'anode. Il suffit dans ce cas de diminuer la tension appliquée au compteur pour retrouver le régime proportionnel. 
On peut même être conduit à réduire l'amplification jusqu'au régime de chambre d'ionisation [14].

b) Train d'impulsions lumineuses rapprochées. - Ce cas se rencontre avec les plasmas produits par des décharges électriques en présence d'une self non négligeable donnant au courant une pseudo-période de une à quelques microsecondes. Il peut aussi se présenter avec l'émission lumineuse des synchrotrons à électrons.

Chaque impulsion lumineuse produit des électrons primaires amplifiés par une avalanche dont les ions, transitant vers la cathode, croisent les électrons primaires créés par les impulsions suivantes. Pour que l'amplification soit constante d'une impulsion à l'autre il faut que les ions apparaissant près de l'anode après une impulsion aient quitté la zone d'amplification avant l'arrivée des électrons primaires dus à l'impulsion suivante. Le temps de transit des ions doit donc être pris en considération et l'on est dans une situation analogue à celle que produit un taux élevé de comptage de photons. Le choix d'une constante de temps du circuit permettant de mesurer chaque impulsion en évitant l'empilement des signaux peut se révéler difficile. Dans le cas d'une décharge électrique on pourra toujours intégrer l'émission sur toute la durée de la décharge. Avec un synchrotron on pourra de même chercher à intégrer l'émission sur un cycle complet d'accélération des électrons ou, si les caractéristiques de la machine le permettent, sur la durée d'un tour des électrons.

Ces remarques montrent que le fonctionnement en intégrateur de flux avec des émissions lumineuses brèves nécessite quelques précautions. Dans ce travail, pour nous assurer de la proportionnalité du signal au nombre de photons, nous avons installé dans le spectrographe un mécanisme porteur de grilles affaiblissantes calibrées qui permettent de faire varier le flux de rayonnement dans des rapports connus et d'ajuster les conditions de fonctionnement du compteur chaque fois que cela est nécessaire.

B) Détermination de L'AMPLification gazeuse. Le nombre très élevé de photons émis par le plasma à chaque tir de laser permet d'obtenir un signal au compteur même avec une faible amplification gazeuse. De ce fait nous avons pu étudier l'amplification entre des valeurs allant de 1 à quelques $10^{3}$ alors que la méthode de comptage des photons, seule applicable aux sources classiques, implique une amplification constamment élevée pour que les signaux restent mesurables en ultraviolet extrême.

Nous avons procédé en mesurant la variation de la hauteur de signal en fonction de la pression gazeuse pour plusieurs valeurs de la tension électrique. Pour ces mesures il faut effectuer un traitement statistique de la réponse du compteur en fonction de la puissance de tir du laser 'selon une méthode qui a déjà été décrite [15]. La figure 2 donne un exemple de la variation de l'amplitude du signal en fonction de la

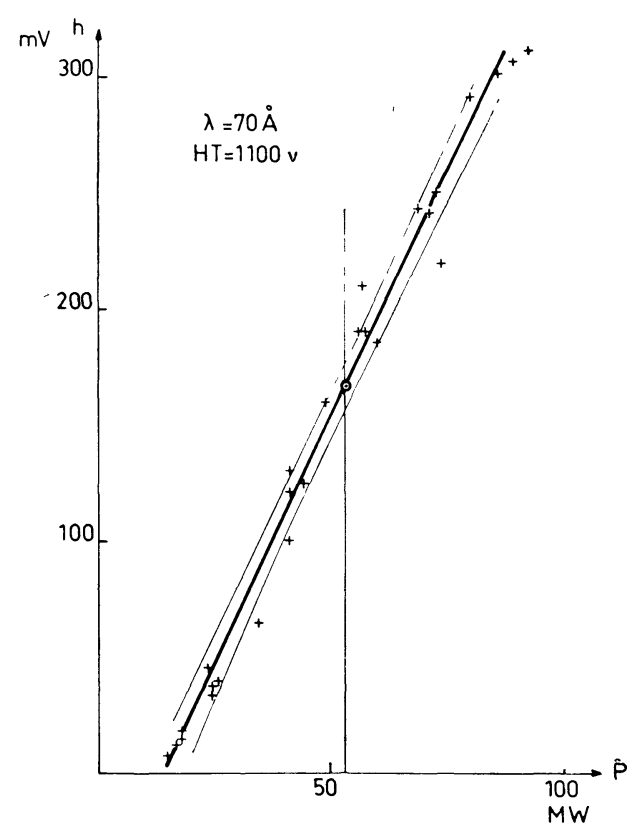

Fig. 2. - Variation de la hauteur du signal en millivolts en fonction de la puissance du laser.

puissance du faisceau laser, c'est-à-dire, en première approximation, de l'intensité du rayonnement $X$ mou pénétrant le compteur. Les arcs d'hyperboles, de part et d'autre de la droite de régression linéaire tracée d'après les résultats de mesures, limitent l'intervalle de confiance de l'amplitude du signal à chaque puissance.

Les mesures sont limitées vers les basses pressions par l'apparition d'une décharge auto-entretenue due à l'émission électronique secondaire de la cathode. Le tableau I donne la pression à laquelle commence cette

\section{TABLEAU I}

Conditions d'amorçage de la décharge auto-entretenue

$\begin{array}{cc}V \text { volt } & p \text { torr } \\ - & - \\ 500 & 1 \\ 700 & 5 \\ 900 & 10 \\ 1100 & 25\end{array}$

décharge en fonction de la tension. La figure 3 représente la hauteur de signal en fonction de la pression, les mesures étant effectuées à $204 \AA$ de longueur d'onde.

L'amplification gazeuse est déduite des courbes de la figure 3 en tenant compte de l'efficacité du compteur qui dépend de la longueur d'onde du rayonnement et en utilisant le résultat d'une mesure absolue effectuée à une pression de 64 torrs de méthane avec une tension de $1000 \mathrm{~V}$ [1]. L'efficacité du compteur a été calculée à partir de données expérimentales sur l'absorption du méthane et de la fenêtre de collodion. L'amplification gazeuse obtenue de la sorte est représentée en fonction de la pression dans le compteur par les courbes en pointillé de la figure 4 . 


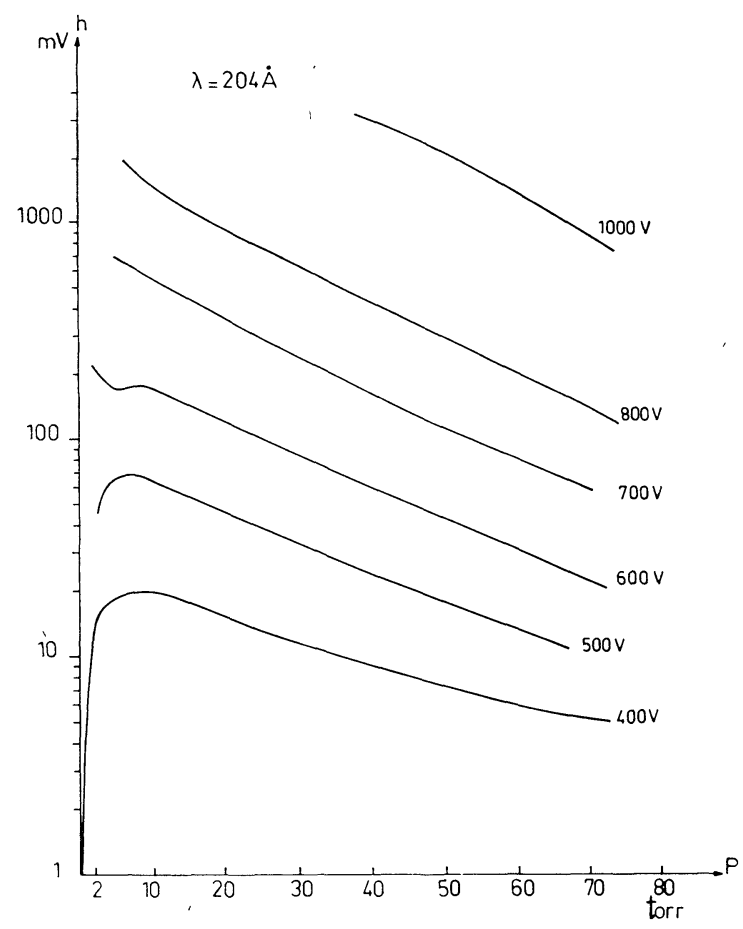

Fig. 3. - Variation de la hauteur du signal en millivolts en fonction de la pression de méthane.

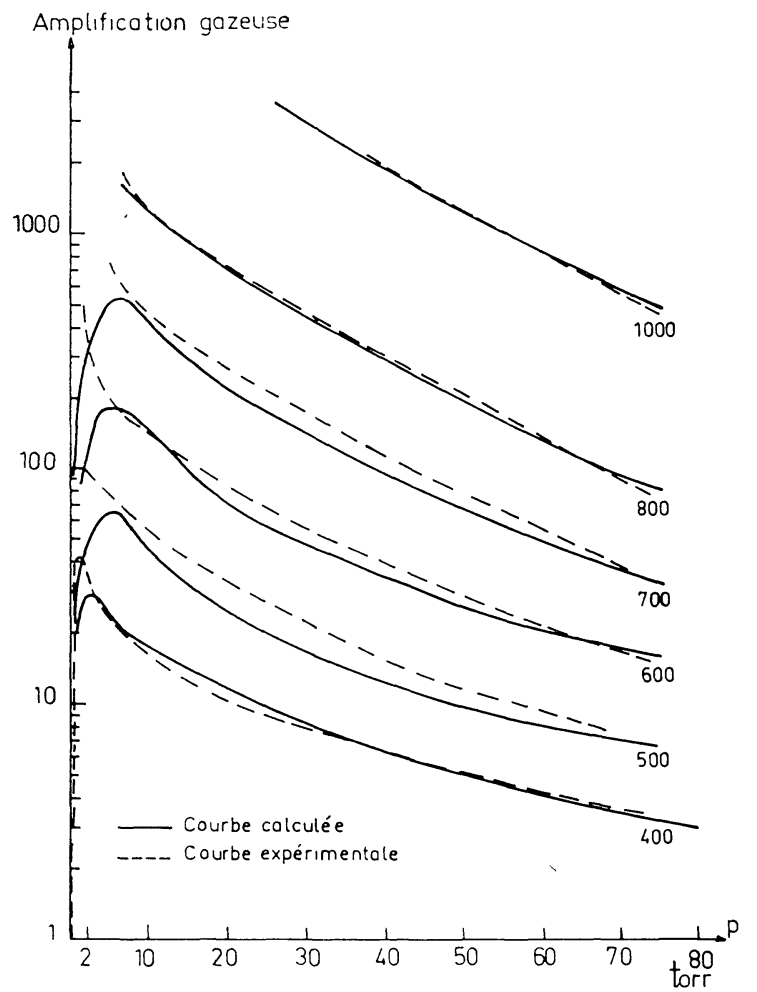

Fig. 4. - Variation de l'amplification gazeuse en fonction de la pression de méthane.

II. Simulation de l'avalanche électronique. Calcul de l'amplification. - Les différentes formules analytiques qui ont été proposées pour le calcul de l'amplification gazeuse utilisent une section efficace d'ionisation par choc électron-molécule définie comme une fonction de $E / p$. Elles sont satisfaisantes en géométrie plane, lorsque $E$ est constant. En géométrie cylindrique, c'est-à-dire pour la plupart des compteurs pratiquement utilisés, leur domaine de validité est limité à $E / p<1000 \mathrm{~V} . \mathrm{cm} /$ torr environ. En effet, au-delà de cette valeur, la distribution d'énergie des électrons ne peut plus être considérée comme constante sur une distance grande par rapport au libre parcours moyen comme l'exige plus ou moins implicitement l'établissement des formules.

De plus l'examen des hypothèses simplificatrices effectuées pour ces calculs montre [16] qu'elles avantagent en fait les électrons d'énergie faible, comparable au seuil d'ionisation. Or après chaque choc ionisant l'un des électrons emporte à lui seul la plus grande partie de l'énergie disponible. De ce fait, si le champ est suffisant pour communiquer une énergie nettement supérieure à l'énergie d'ionisation à l'électron dans l'intervalle séparant deux chocs ionisants, les électrons de grande énergie sont nombreux dans l'avalanche. Cela se produit nécessairement au voisinage de l'anode lorsque $E / p$ atteint une valeur suffisante. Les formules classiques sont alors en défaut.

Non seulement les valeurs calculées s'écartent des valeurs expérimentales, mais elles croissent de façon monotone lorsque la pression diminue, ce qui est en contradiction flagrante avec le fait qu'il ne saurait y avoir d'amplification lorsqu'il n'y a pas de gaz. On doit s'attendre à ce que l'amplification passe par un maximum lorsque la pression décroît et tende vers 1 quand la pression s'annule. C'est en effet ce que montre la courbe expérimentale établie à $400 \mathrm{~V}$ dans notre travail (Fig. 4).

La difficulté d'exprimer analytiquement l'amplification conduit à rechercher une méthode de simulation de l'avalanche en ordinateur. Des résultats dans ce sens ont été obtenus avec une méthode de Monte Carlo [17] dans laquelle chaque électron était suivi individuellement et chacune de ses interactions calculée sur une base probabiliste. Comme l'avalanche peut compter jusqu'à $10^{5}$ électrons dont chacun subit des milliers d'interactions cette méthode nécessiterait dans notre cas un volume énorme de calcul. Aussi, plutôt que de tenir compte dans le calcul de chaque interaction, nous avons tenté de définir à l'avance quelles étaient les interactions les plus importantes et de les introduire sous une forme qui respecte globalement l'évolution de l'avalanche.

A) HyPothèSES GÉNÉRALES. - Il y a lieu de distinguer trois types de chocs électron-molécule : les chocs élastiques, les chocs inélastiques non ionisants (excitation électronique, vibration, rotation) et les chocs ionisants. Les pertes d'énergie par chocs élastiques sont proportionnelles au rapport des masses électroniques et moléculaires. On peut les négliger par rapport aux autres pertes. Les autres approximations importantes sont les suivantes.

a) Le modèle est unidimensionnel. - Dans les chocs élastiques la diffusion des électrons n'est isotrope que 
pour les énergies inférieures à $1 \mathrm{eV}$ environ. A quelques dizaines d'électrons-volts et plus la diffusion a lieu vers l'avant [18], [19] sous un angle d'autant plus faible que l'énergie est plus grande. Dans les chocs inélastiques la déviation des électrons est en moyenne d'autant plus faible que la fraction d'énergie perdue est petite. Dans l'avalanche les électrons ont une énergie suffisante et les pertes d'énergie sont assez petites pour que l'on puisse supposer que la diffusion a lieu vers l'avant sous un angle généralement faible. Nous avons donc admis qu'un calcul effectué le long d'un rayon $\mathrm{du}$ cylindre pourrait donner une bonne approximation.

b) Les pertes d'énergie par excitation sont introduites sous forme de valeurs moyennes déduites de l'expérience. - Un électron gagne de l'énergie sous l'action du champ électrique et en perd dans des chocs inélastiques non ionisants avec les molécules de gaz, c'est-à-dire en excitant celles-ci. Ces pertes retardent le moment où son énergie est suffisante pour provoquer une ionisation. Globalement il s'agit d'un freinage dont l'évaluation est primordiale pour le calcul de l'amplification. En toute rigueur il faudrait tenir compte des sections efficaces correspondant à chaque type d'excitation. Mais dans le problème qui nous occupe le paramètre important est le nombre de molécules rencontrées par un électron par unité de longueur sur son parcours et on peut envisager de définir une perte moyenne par choc englobant des sections efficaces de différentes sortes dont la variation en fonction de l'énergie a la même allure générale.

Partant de la courbe de section efficace totale du méthane, incluant toutes les interactions élastiques et inélastiques [20] et des courbes de sections efficaces élastiques et d'ionisation [18], [21], nous avons obtenu par soustraction une section efficace d'excitation $\sigma(\varepsilon)$ en fonction de l'énergie $\varepsilon$. Pour les chocs élastiques nous avons utilisé les données de l'argon [22] dont la théorie prévoit qu'elles s'écartent peu de celles du méthane. La figure 5 représente $\sigma(\varepsilon) / \varepsilon$ en fonction

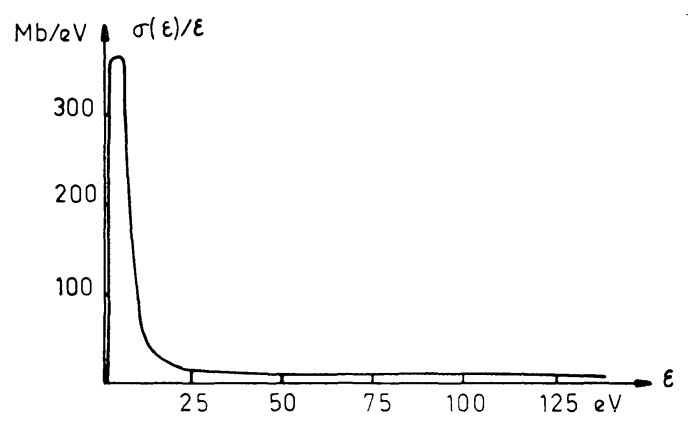

FIG. 5. - Section efficace globale d'excitation $\sigma(\varepsilon)$ : en ordonnée $\sigma(\varepsilon) / \varepsilon ;$ en abscisse l'énergie $\varepsilon$.

de $\varepsilon$. Au cours du calcul de l'avalanche on évaluera l'énergie d'un électron et on déduira de cette courbe la perte d'énergie correspondant au choc survenant à cette énergie. Le nombre de chocs, proportionnel à la pression, déterminera finalement la perte d'énergie sur un parcours donné.

c) L'inégalité de répartition d'énergie entre les deux électrons consécutifs à un choc ionisant confère à la distribution d'énergie son allure générale. - La distribution d'énergie des électrons dans l'avalanche joue un rôle important puisque toutes les sections efficaces en dépendent. Mais il n'est pas nécessaire que l'énergie de chaque électron soit connue avec le plus de précision possible. Il suffit que l'allure générale de la distribution soit respectée. Or celle-ci est déterminée par le fait établi expérimentalement [18], [23], [24], [25] qu'en moyenne, après un choc ionisant, un des électrons possède une énergie cinétique faible vis-à-vis de l'autre. Cette inégalité de répartition donne une distribution d'énergie à deux maximums, l'un à quelques électrons-volts, l'autre à plusieurs dizaines d'électronsvolts.

Soit $\beta$ le pourcentage de l'énergie emportée par l'un des deux électrons et $1-\beta$ le pourcentage correspondant à l'autre. La valeur de $\beta$ joue un rôle critique dans le calcul de l'amplification comme le montre la courbe de la figure 6 où est portée, en coordonnées, l'amplifi-

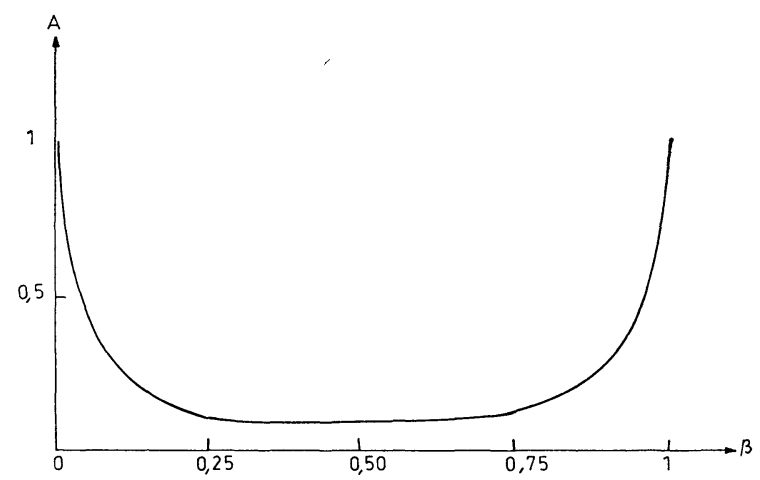

Fig. 6. - Calcul de l'amplification gazeuse A en unités relatives en fonction de la proportion $\beta$ d'énergie emportée par l'électron " éjecté ».

cation calculée pour une tension de $800 \mathrm{~V}$ et une pression de 50 torrs. La variation est rapide pour les valeurs de $\beta$ voisines de 0 et de 1 . Or, pour un faisceau d'électrons incidents de $21 \mathrm{eV}$ dans l'hydrogène, on peut déduire de la courbe de distribution en énergie des électrons arrachés [25] que ceux-ci emportent environ $6 \%$ de l'énergie disponible. En l'absence d'expérience dont on puisse déduire une valeur moyenne de $\beta$ dans le cas du méthane, nous avons donc adopté la valeur $\beta=0,06$.

d) Le dénombrement des chocs ionisants utilise une valeur moyenne de l'énergie d'ionisation. - Le choix d'une méthode de calcul non probabiliste exclut la possibilité d'utiliser une courbe expérimentale de section efficace pour déterminer, non plus une simple perte d'énergie comme dans le cas des excitations, mais la production ou non d'une ionisation par un électron ayant atteint une certaine énergie. L'énergie de chaque 
1 : électron d'énergie $E$ part d'un point situé à une distance $k_{1} . l(p)$ de la coque et se déplace vers le fil.

Initialisation de $E$ et de $k_{1}$.

\section{Les électrons ont-ils atteint} le fil ?

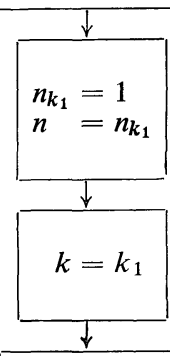

$l(p)$ 《pas » du calcul en $\mathrm{cm}$ à la pression $p$

$n_{k_{1}} \quad$ nombre total d'électrons créés à la distance $k_{1} \cdot l(p)$,

$E_{\text {après }}$ énergie d'un électron après un choc ionisant ou non,

$E_{\text {avant }}$ énergie d'un électron à l'instant précédant juste le choc,

$E_{\mathrm{m}} \quad$ énergie moyenne des électrons ionisants,

$\beta \quad$ pourcentage de l'énergie totale emportée par l'électron éjecté,

$\chi=1-\gamma \sigma(\varepsilon) / \varepsilon$ «facteur de freinage $»$,

$\varepsilon_{\mathrm{i}} \quad$ énergie d'ionisation $(13 \mathrm{eV})$,

$A$ amplification gazeuse.

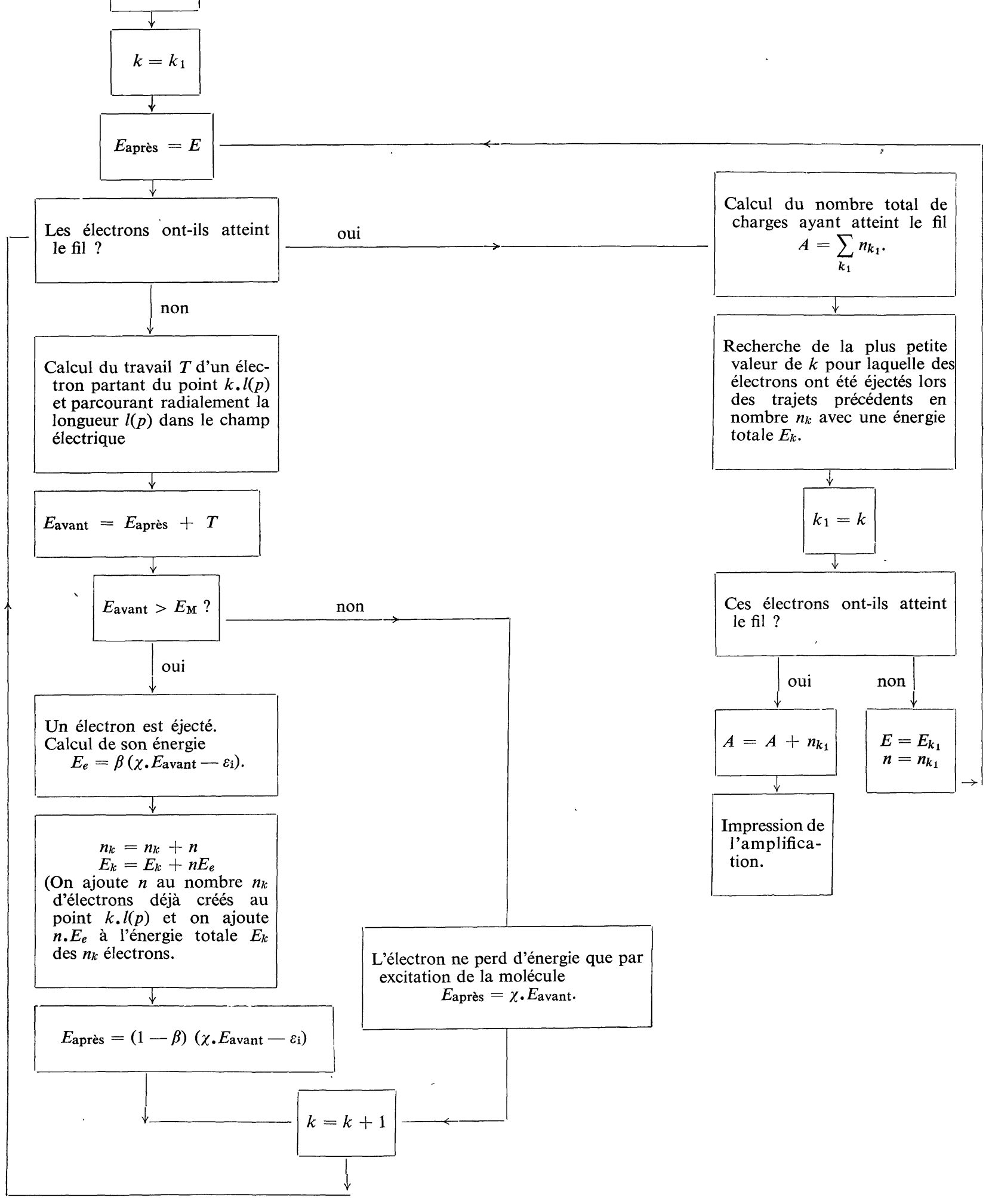

FIG. 7. - Organigramme du calcul d'amplification. 
électron est donc comparée périodiquement à une énergie $E_{\mathrm{m}}$ considérée comme valeur moyenne des énergies auxquelles les ionisations ont effectivement lieu et l'on admet qu'il y a ionisation chaque fois que l'énergie atteint la valeur $E_{\mathrm{m}}$.

B) Simulation de L'Avalanche et paramètres AJUSTABLES. - L'organisation du calcul est représentée schématiquement par le diagramme de la figure 7 . Le pas du calcul est défini comme suit : chaque fois qu'un trajet élémentaire égal à ce pas est parcouru par un électron «quelque chose» se passe qui est soit un freinage, soit une ionisation; si $l$ est ce trajet à la pression de 1 torr, on prend $l(p)=l / p$ à la pression $p ; l$ est traité comme un paramètre ajustable alors que $p$ est une variable.

Le programme calcule le gain d'énergie le long de $l(p)$ et la perte d'énergie à l'extrémité de $l(p)$. Pour un choc non ionisant $\left(\varepsilon<E_{\mathrm{m}}\right)$ la perte est évaluée au moyen du facteur de freinage

$$
\chi=1-\gamma \frac{\sigma(\varepsilon)}{\varepsilon}
$$

où $\gamma$ est un second paramètre ajustable. Si le choc est ionisant $\left(\varepsilon \geqslant E_{\mathrm{m}}\right)$ la perte est égale à l'énergie d'ionisation $\varepsilon_{\mathrm{i}}=13 \mathrm{eV}$ et l'énergie restante est répartie, pour les raisons déjà exposées, dans la proportion de $6 \%$ pour l'électron "éjecté » et de $94 \%$ pour l'électron "incident». Le troisième paramètre ajustable est l'énergie moyenne d'ionisation $E_{\mathrm{m}}$.

Les trajets élémentaires $l(p)$ sont indicés. La valeur de l'indice fixe la distance à l'anode du petit trajet considéré où s'achèvera le calcul. A chaque valeur de l'indice correspond aussi une mémoire dans laquelle est stocké l'électron éventuellement créé par ionisation à cette distance. Le parcours du premier électron charge donc un certain nombre de mémoires. Ce parcours terminé, le programme cherche la mémoire non vide la plus éloignée de l'anode et le calcul recommence. Il arrive rapidement qu'une mémoire contienne plusieurs électrons dont les énergies s'écartent d'ailleurs peu d'une valeur moyenne de quelques électronsvolts. On remplace alors les $N$ électrons de cette mémoire par un électron fictif ayant pour énergie cette valeur moyenne et l'on multiplie par $N$ les effets de cet électron sur la création de nouveaux électrons; le nombre de calculs qui seraient nécessaires sans cette approximation est ainsi chaque fois divisé par $N$. Lorsque toutes les mémoires sont vides sauf celle qui est à proximité immédiate de l'anode, l'avalanche est terminée et il ne reste qu'à compter les électrons parvenus à l'anode.

Il va de soi que la durée du calcul dépend fortement de la valeur donnée à la pression. A 100 torrs, elle ne dépasse pas $2 \mathrm{~s}$ sur IBM 360. Elle est couramment comprise entre $1 \mathrm{~s}$ et $2 \mathrm{~s}$.

C) Discussion des RéSultats. - Le modèle présenté ici dépend de trois paramètres ajustables, $l, \gamma$,
$E_{\mathrm{m}}$, et de deux variables qui sont la pression de gaz dans le compteur et la tension appliquée entre l'anode et la cathode cylindrique. La valeur de ce modèle dépend de son aptitude à rendre compte de l'ensemble des résultats expérimentaux pour un seul groupe de valeurs numériques des paramètres ajustables.

Les figures 4 et 8 permettent de comparer les résultats expérimentaux et les courbes théoriques calculées avec :

$$
\begin{aligned}
l & =0,011 \mathrm{~cm} \\
\gamma & =10^{-4} \mathrm{eV} / \mathrm{Mb} \\
E_{\mathrm{m}} & =135 \mathrm{eV} .
\end{aligned}
$$

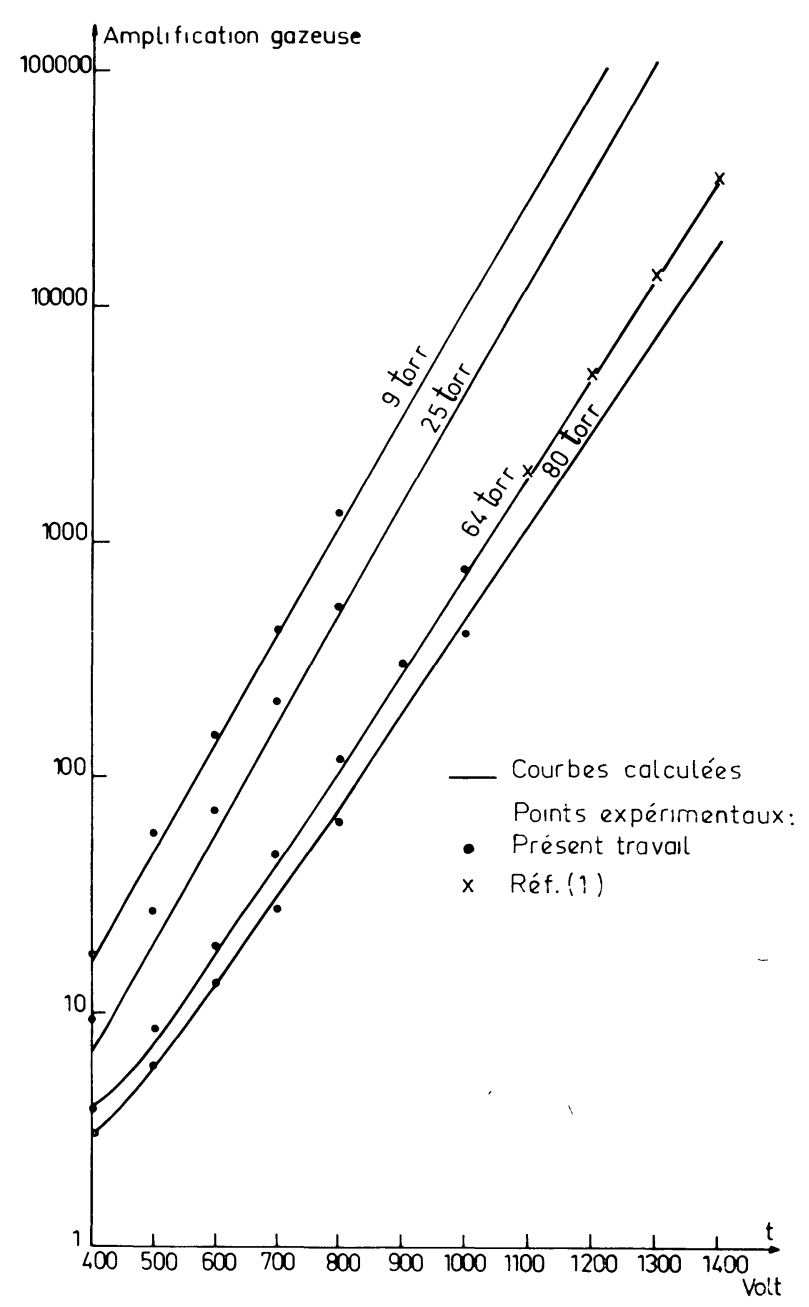

FIG. 8. - Variation de l'amplification gazeuse en fonction de la tension.

La figure 4 donne la variation de l'amplification en fonction de la pression pour plusieurs tensions et la figure 8 , la variation de l'amplification en fonction de la tension. Dans les deux cas, les courbes théoriques sont en traits pleins. Comme on le constate, l'accord est bon non seulement dans le domaine de pression et de tension que nous avons étudié, mais aussi pour des valeurs expérimentales obtenues par d'autres auteurs [1].

Un désaccord n'apparaît, figure 4, que pour les 
pressions inférieures à 10 torrs lorsque la tension dépasse $400 \mathrm{~V}$, c'est-à-dire à proximité de l'apparition de la décharge auto-entretenue (tableau I). Ce désaccord était attendu puisque le modèle n'inclut pas les photo-électrons émis par la cathode dont le nombre augmente rapidement avant l'apparition de la décharge. Pour les courbes à $400 \mathrm{~V}$ (Fig. 4) la décharge n'ayant pas lieu, on peut comparer directement les maximums expérimentaux et théoriques d'amplification. La petite différence observée dans la position et dans la hauteur des maximums est également attribuable aux photoélectrons.

Nous sommes donc fondés à conclure que le modèle donne une représentation correcte du phénomène expérimental d'avalanche pour des valeurs de $E / p$ allant jusqu'à quelques $10^{4} \mathrm{~V} / \mathrm{cm}$.torr c'est-à-dire dix fois supérieures à celles que permettaient les autres calculs.

Une brève discussion des valeurs numériques des paramètres permet, pour finir, de donner des indications sur leur signification physique. La remarque la plus intéressante concerne la valeur de $\gamma$. On peut en effet associer à $\gamma$ une perte d'énergie moyenne $\bar{\varepsilon}_{e}$ par choc au moyen de la relation [16]

$$
\gamma_{\mathrm{eV}} / \mathrm{Mb}=3,54 \times 10^{-4} \times l_{\mathrm{cm}} \times \bar{\varepsilon}_{e_{\mathrm{eV}}}
$$

qui donne ici $\bar{\varepsilon}_{e}=0,26 \mathrm{eV}$, valeur très voisine des énergies des bandes de vibration du méthane. Or, on sait que les sections efficaces d'excitation vibrationnelle sont supérieures de deux ordres de grandeur aux sections efficaces d'excitation électronique. Le modèle rend donc compte de ce que le freinage doit, en effet, provenir essentiellement de l'excitation des vibrations moléculaires.

A propos de la valeur $0,011 \mathrm{~cm}$ obtenue pour $l$, il est à noter que le libre parcours moyen déduit de la courbe de section efficace totale pour des électrons de quelques dizaines d'électrons-volts [20] est d'environ $0,03 \mathrm{~cm}$, donc du même ordre de grandeur que $l$. La différence subsistant entre ces deux valeurs s'explique probablement par le fait que le modèle est unidimensionnel et que, de plus, la variation rapide du champ au voisinage de l'anode interdit d'assimiler complètement la grandeur $l$ à un libre parcours moyen.
De la valeur $135 \mathrm{eV}$ obtenue pour $E_{\mathrm{m}}$ nous dirons simplement qu'elle suggère que les électrons ionisants sont en moyenne un peu plus rapides qu'on aurait pu l'attendre. En effet le seuil d'ionisation est à $13 \mathrm{eV}$ et le maximum de la courbe de section efficace est atteint vers $70 \mathrm{eV}$ [21]. Mais la section efficace décroît assez lentement vers les grandes énergies et, à $135 \mathrm{eV}$, elle n'a diminué que de $15 \%$. L'hypothèse que $E_{\mathrm{m}}$ est une énergie moyenne d'ionisation n'entre donc pas en contradiction avec les données expérimentales sur le méthane.

Nous avons réalisé ce modèle dans le but pratique de prévoir des possibilités plus étendues de fonctionnement des compteurs proportionnels. La figure 9

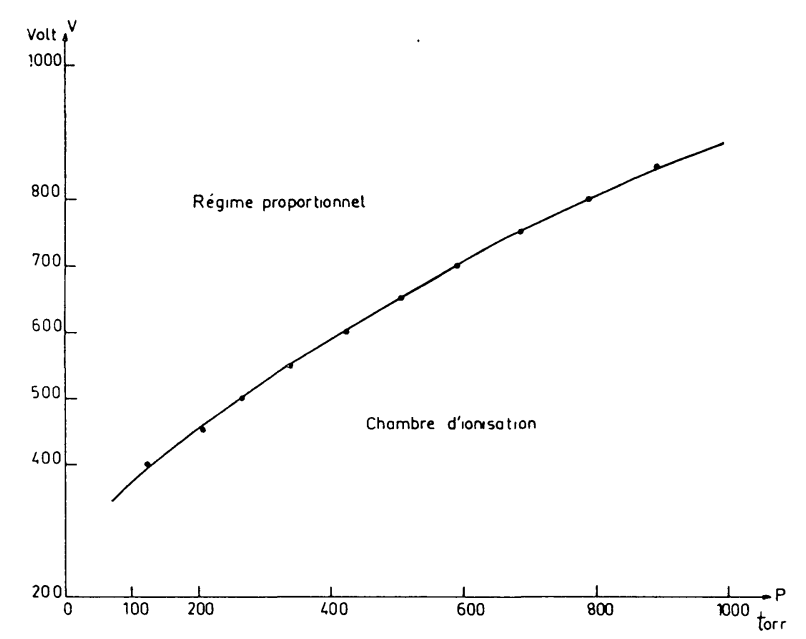

FIG. 9. - Tension (en ordonnées) à laquelle commence l'amplification pour une pression donnée (en abscisses).

donne un exemple de son utilisation pour la détermination des tensions et des pressions pour lesquelles commence l'amplification, c'est-à-dire du passage du régime de chambre d'ionisation au régime proportionnel.

Remerciements. - Les auteurs expriment leurs remerciements au Professeur Y. Cauchois pour l'intérêt qu'elle a pris à leur travail et l'aide qu'ils en ont reçue.

\section{Bibliographie}

[1] Lukirski (A. P.), Ershov (O. A.), Brytov (I. A.). - Bull. Acad. Sci., USSR, Physical series, 1963, 27, 798.

[2] Lukirski (A. P.), Ershov (O. A.), Brytov (I. A.). - Inst. and Exp. Techn., 1965, 5, 1083.

[3] Dhez (P.). - Thèse, Orsay 1971.

[4] Von ENGEL (A.). - Gas descharges I-Encyclopedia of Physics, 1956, 21, 504.

[5] Rose (M. E.), Korff (S. A.). - Phys. Rev., 1941, $59,850$.

[6] Campion (P. J.). - Int. J. of Appl. Rad. and Isot., 1968, 19, 219.

[7] William (A. W.), Sara (R. I.). - Int. J. of Appl. Rad. and Isot., 1962, 13, 229.
[8] Gold (R.), Benett (E. F.). - Phys. Rev., 1966, 147, 201.

[9] Specht (M. J.), Ambuster (P.). - Nucleonik, 1965, $7,8$.

[10] Dhez (P.), Jaegle (P.), Leach (S.), Velche (M.). - J. Appl. Phys., 1969, 40, 2545.

[11] Dhez (P.) Jaegle (P.). - Revue. Phys. Appl., 1968, 3, 275.

[12] Charles (M. W.), Cooke (B. H.). - Nucl. Inst. and Methods, 1968, 61, 31.

[13] Jeagle (P.). - Thèse, Paris 1965. C. R. Acad. Sci. Paris, 1964, 259, 533. C. R. Acad. Sci. Paris, 1964, 259, 4556.

[14] Dhez (P.), Jaegle (P.). - C. R. Acad. Sci. Paris, 1966, 262, 1432. 
[15] Dhez (P.), Jaegle (P.). - Colloque CNRS. « Processus électroniques simples et multiples du domaine $\mathrm{X}$ et X-UV » à paraître au $J$. Physique, 1972.

[16] Cukier (M.). - Thèse de $3^{\mathrm{e}}$ cycle, Orsay 1971.

[17] Thomas (R. W. L.), Thomas (W. R. L.). - J. Phys. B. (Atom. Mol. Phys.), 1969, 2, 562.

[18] Papoular (R.). - Phénomènes électriques dans les gaz, Ed. Dunod, 1963.

[19] Hugues (A. C.), McMillen (J. H.). - Phys. Rev., 1932, 39, 585.
[20] Kollath (R.). - Corpuscules and radiations in matter II, Encyclopedia of Physics, 1959, 10, 34

[21] AdamczyK (B.), Boerboom (A. J.), Schram (B. L.), Kisteimaker (J.). - J. Chem. Phys., 1966, 44, 4640.

[22] Cragg (J. D.), Massey (H. S. W.). - Atoms III, Molecules I, Encyclopedia of Physics, 1969, 37/1, 332.

[23] Tate (T.), Palmer (R. R.). - Phys. Rev., 1932, $39,585$.

[24] Goodrich (M.). - Phys. Rev., 1937, 52, 259.

[25] Mott (N. F.), Massey (H. S. W.). - The theory of atomic collisions, 491, Oxford, 1969. 